\title{
Das Marktortprinzip in der DS-GVO
}

Die Durchsetzung datenschutzrechtlicher Vorgaben erfordert stets, dass die Vorschriften auf einen gegebenen Sachverhalt überhaupt anwendbar sind. Jedes Gesetz muss daher seinen eigenen Anwendungsbereich in persönlicher, sachlicher und räumlicher Hinsicht festlegen, es sei denn, dieser ergibt sich bereits aus den Umständen, z. B. aufgrund seiner systematischen Stellung innerhalb der Rechtsordnung.

Insbesondere die Kriterien, die die Europäische Datenschutzrichtlinie (DS-RL) für die räumlichen Anwendungsregeln der mitgliedstaatlichen Datenschutzvorschriften vorsah, waren eine Ursache des oft beklagten Durchsetzungsdefizits dieses Regelwerks. ${ }^{1}$ Datenverarbeiter, die keine Niederlassung in einem EU-Mitgliedstaat betreiben, sollte das nationale Recht gem. Art. 4 Abs. 1 lit. c DS-RL nur dann erfassen, wenn sie für die in Frage stehende Datenverarbeitung auf Mittel zurückgriffen, welche sich in dem betreffenden EU-Mitgliedstaat befanden. Der Begriff des Mittels wurde dabei immer weiter ausgedehnt, sodass darunter zuletzt sogar auch kleinste Programme und Anwendungen wie Cookies gefasst wurden. ${ }^{2}$ Der eigentlichen Problematik, dass sich Datenverarbeiter durch Verlegung ihrer Rechenzentren in Drittländer den europäischen Datenschutzregelungen entziehen können, obwohl sie ihre Dienste auf EU-Bürger abstimmen, half jedoch auch eine derart weite, nah an der Wortlautgrenze verlaufende Auslegung nur bedingt ab.

Der Gesetzgeber nutzte die europäische Datenschutzreform, um Verantwortliche aus Drittländern deutlicher als bisher in den Adressatenkreis der Datenschutzvorschriften mit einzuschließen. Er bediente sich dabei eines ursprünglich aus dem Wettbewerbsrecht stammenden Prinzips: des Marktortprinzips. Hiernach wird das anzuwendende Recht - wie der Name vermuten lässt - nach dem Marktort, d. h. nach dem Ort, wo ein Wettbewerber auf seine Kunden einwirkt, ${ }^{3}$ bestimmt. Verantwortliche ohne Niederlassung in der EU sind nunmehr gemäß Art. 3 Abs. 2 lit. a DS-GVO auch dann den europäischen Regelungen unterworfen, wenn ihre Datenverarbeitungstätigkeit mit dem Anbieten von Waren oder Dienstleistungen an betroffene Personen in der EU zusammenhängt. Damit führt der Gesetzgeber einen bereits vom EuGH eingeschlagenen Pfad fort. Dieser hatte in seiner Google Spain-Entscheidung für die Anwendung der DS-RL bereits eine Ausrichtung des Verantwortlichen auf einen EU-Mitgliedstaat genügen lassen und so das Marktortprinzip bereits vor der Gesetzesreform praktisch eingeführt. ${ }^{4}$ Allerdings nutzte der EuGH diese Auslegung, um das Unterhalten einer Niederlassung i. S. v. Art. 4 Abs. 1 lit. a DS-RL ${ }^{5}$ zu begründen, was zu einer starken Ausdehnung dieses Begriffs führte. Die Übertragung dieses Ansatzes auf Verantwortliche ohne Niederlassung stellt diese Praxis somit ausdrücklich auf eine rechtliche Grundlage.

Die Wahl eines marktortbasierten Anknüpfungspunktes bietet eine angemessene und moderne Antwort auf die Problema-

\footnotetext{
1 Siehe u. a. Weichert, NStZ 1999, 490 (492).

2 Kroschwald, Informationelle Selbstbestimmung in der Cloud, 2016, S. $153 \mathrm{ff}$.

3 Köhler/Bornkamm/Köhler, UWG, Einleitung Rn. 5.5.

4 EuGH, 13.5.2014 - C-131/12, Google Spain, DuD 2014,559 (562).
}

5 Entspricht im Wesentlichen dem Art. 3 Abs. 1 DS-GVO. tik, dass die Lokalisierung einzelner Datenverarbeitungsvorgänge durch Technologien wie Cloud Computing nicht nur schwieriger, sondern auch bedeutungsloser wird. Der Rechtsanwender muss stattdessen künftig lediglich ermitteln, ob ein „Anbieten“ stattfindet. Zur Auslegung dieses Terminus liefert EG 23 einige Hinweise. Danach kann vor allem die Verwendung einer Sprache oder Währung, die in einem oder mehreren Mitgliedstaaten verwendet wird, aber auch die Erwähnung von Kunden oder Nutzern in der EU darauf hindeuten, dass der Verantwortliche beabsichtigt, seine Waren und Dienstleistungen dort anzubieten. Es ist dabei unerheblich, ob der Verantwortliche ein Entgelt erhebt. Darüber hinaus kann zur näheren Bestimmung der Marktortregel auf die Auslegung des EuGH zu dem verbraucherrechtlichen Begriff des „Ausrichtens“ zurückgegriffen werden. Der EuGH nennt als mögliche Indizien auch die Verwendung einer Top-Level-Domain eines Mitgliedstaates. ${ }^{6}$ Dieses Merkmal dürfte besonders im E-Commerce-Bereich regelmäßig ein wichtiges Anzeichen für ein Abstimmen des $\mathrm{Ge}$ schäftstreibenden auf den europäischen Markt sein.

Fraglich ist derzeit allerdings noch, wie ausgeprägt die subjektiven Elemente seitens des Verantwortlichen sein müssen. EG 23 setzt zunächst nur eine Intention des Verantwortlichen voraus, seine Waren oder Dienstleistungen auf dem Gebiet der EU anzubieten. ${ }^{7}$ Obgleich der EuGH im Verbraucherrecht auch ein aktives Tun seitens des Unternehmers verlangt, durch welches neue Kunden auch faktisch gewonnen werden, ${ }^{8}$ erscheint eine Übertragung auf das Datenschutzrecht an dieser Stelle nicht zweckgemäß. Der tatsächliche Gewinn neuer Kunden ist hier weniger entscheidend, da eine Verarbeitung personenbezogener Daten regelmäßig unabhängig von einer vertraglichen Beziehung zwischen Verantwortlichem und Betroffenem stattfindet.

Für die Implementierung von Cookies sowie anderer Programme und Anwendungen zur Nachverfolgung von Internetaktivitäten schuf der Gesetzgeber mit Art. 3 Abs. 2 lit. b DS-GVO eine eigene Anwendungsvorschrift. Ein Verantwortlicher fällt danach auch dann unter die DS-GVO, wenn seine Datenverarbeitung mit der Beobachtung betroffener Personen - vor allem online ${ }^{9}-$ im $\mathrm{Zu}$ sammenhang steht. Auffallend ist, dass hierfür keinerlei subjektive Elemente, z. B. in Form einer Beabsichtigung, verlangt werden. Dieser Umstand führt zu einer sehr weiten Regelung, bei welcher der Bezug zur EU mitunter auch nur minimal ausfallen kann. ${ }^{10}$

Insgesamt stellt das Marktortprinzip einen sachgerechten Weg dar, um Datenverarbeitungsvorgänge im EU-Ausland in den Schutz der DS-GVO zu stellen. Sie vermeidet eine Überdehnung des Niederlassungsbegriffs und schafft letztendlich mehr - wenn auch keine vollkommene ${ }^{11}-$ Rechtssicherheit.

\footnotetext{
6 EuGH, Urt. v. 7.12.2010 - C-585/08, Pammer und Alpenhof, EuZW 2011, 98 (104).

7 "[Es] können andere Faktoren [...] darauf hindeuten, dass der Verantwortliche beabsichtigt, den Personen in der Union Waren oder Dienstleistungen anzubieten".

8 EuGH, Urt. v. 7.12.2010 - C-585/08, Pammer und Alpenhof, EuZW 2011, 98 (102 f.).

9 Vgl. EG 24.

10 Klar, DuD 2017, 533 (536).

11 Zu einigen Unklarheiten des Art. 3 DS-GVO s. den Beitrag v. Golland (in diesem Heft).
} 